\title{
Update on the management of cirrhosis - focus on cost-effective preventative strategies
}

This article was published in the following Dove Press journal:

ClinicoEconomics and Outcomes Research

10 April 2013

Number of times this article has been viewed

\author{
Guy W Neff' \\ Nyingi Kemmer ${ }^{\prime}$ \\ Christopher Duncan² \\ Angel Alsina' \\ 'Tampa General Medical Group, \\ Tampa, FL, ${ }^{2}$ Highline Gastroenterology, \\ Seattle, WA, USA
}

\begin{abstract}
Cirrhosis is a chronic liver disease stage that encompasses a variety of etiologies resulting in liver damage. This damage may induce secondary complications such as portal hypertension, esophageal variceal bleeding, spontaneous bacterial peritonitis, and hepatic encephalopathy. Screening for and management of these complications incurs substantial health care costs; thus, determining the most economical and beneficial treatment strategies is essential. This article reviews the economic impact of a variety of prophylactic and treatment regimens employed for cirrhosis-related complications. Prophylactic use of $\beta$-adrenergic blockers for portal hypertension and variceal bleeding appears to be cost-effective, but the most economical regimen for treatment of initial bleeding is unclear given that cost comparisons of pharmacologic and surgical regimens are lacking. In contrast, prophylaxis for spontaneous bacterial peritonitis cannot be recommended. Standard therapy for spontaneous bacterial peritonitis includes antibiotics, and the overall economic impact of these medications depends largely on their direct cost. However, the potential development of bacterial antibiotic resistance and resulting clinical failure should also be considered. Nonabsorbable disaccharides are standard therapies for hepatic encephalopathy; however, given their questionable efficacy, the nonsystemic antibiotic rifaximin may be a more cost-effective, long-term treatment for hepatic encephalopathy, despite its increased direct cost, because of its demonstrated efficacy and prevention of hospitalization. Further studies evaluating the cost burden of cirrhosis and cirrhosis-related complications, including screening costs, the cost of treatment and maintenance therapy, conveyance to liver transplantation, liver transplantation success, and health-related quality of life after transplantation, are essential for evaluation of the economic burden of hepatic encephalopathy and all cirrhosis-related complications.
\end{abstract}

Keywords: hepatic encephalopathy, maintenance of remission, rifaximin, lactulose, cost

\section{Introduction}

Cirrhosis is a chronic liver disease with a multifactorial etiology (eg, chronic hepatitis viral infection, alcoholic liver disease, nonalcoholic fatty liver disease) that results in liver damage. ${ }^{1}$ Resistance to liver blood flow because of fibrosis leads to portal hypertension (PH) and the formation of portosystemic collaterals, ${ }^{2}$ which reduce blood flow through the liver ${ }^{3}$ and lead to the accumulation of toxins in the systemic circulation. Symptoms of cirrhosis occur along a continuum, from no symptoms in the early stages of the disease to severe complications resulting from $\mathrm{PH}$ (eg, esophageal variceal bleeding [EVB]) and reduced toxin clearance (eg, hepatic encephalopathy [HE]).

The primary goals of therapy for cirrhosis are to manage patients' symptoms and prevent the occurrence of cirrhosis-related complications until liver transplantation can 
be performed. ${ }^{2,4}$ The standard of care strategy includes appropriate screening for and treatment of complications secondary to cirrhosis (eg, $\mathrm{PH}, \mathrm{EVB}$, spontaneous bacterial peritonitis [SBP], and HE). ${ }^{2}$ Although the incidence of these disorders has remained relatively stable in recent years, increases in the cost associated with hospitalization, screening, and treatment of these cirrhosis-related complications have raised concerns about the economic burden of these disorders. This review briefly discusses the economic impact of cirrhosis and highlights the health care cost burden of cirrhosis-related complications with a particular emphasis on HE.

\section{Economic impact of cirrhosis}

In the general population worldwide, up to $10 \%$ of people are estimated to have liver cirrhosis, although precise estimation is confounded because the disease may remain undiagnosed until related complications (eg, ascites, edema, bruising, encephalopathy) become apparent. ${ }^{5}$ Cirrhosis is one of the leading causes of death worldwide, resulting in morbidity, a significant reduction in health-related quality of life (HRQOL), and a direct cost (excluding hepatitis $\mathrm{C}$ virus $[\mathrm{HCV}]$ ) of approximately $\$ 1.4$ billion in the US. The main causes of liver cirrhosis are $\mathrm{HCV}$, hepatitis B virus, and alcohol consumption, suggesting that the disease may be preventable. Indeed, several practices have been implemented to reduce the risk of hepatitis infections, including screening of blood donors and pregnant women for infection, vaccination programs for infants and high-risk populations, and the establishment of infection control practices in hospitals. ${ }^{6}$ Unfortunately, the cost of some of these programs may not justify their implementation despite the fact that the effects of chronic infection (eg, cirrhosis) could be avoided. ${ }^{7}$ In addition, despite a reduction in new infections, the cost of managing aging patients with chronic liver disease may be substantial. For example, in 2009 the per-patient cost of managing chronic $\mathrm{HCV}$ infection was projected to increase 3.5 times over the next 20 years. ${ }^{8}$

Currently, the management of patients with cirrhosis focuses on treating underlying diseases (eg, $\mathrm{HCV}$, hepatitis B virus $)^{2}$, and associated symptoms, while maintaining patients' health until liver transplantation is possible. ${ }^{4}$ In general, according to the Healthcare Cost and Utilization Project (HCUP), the number of hospital discharges for chronic liver disease and cirrhosis from 2002 until 2010 has increased from roughly 400,000 to 525,000 (Figure 1). ${ }^{9}$ The cost of hospitalization decreased slightly from 2005 to 2006 , but still far exceeded that reported in 1993. The substantial increase in hospitalization costs may be attributable to inflation of overall health care expenditures and alterations in therapeutic regimens for the treatment of cirrhosis-related diseases (eg, $\mathrm{HCV}$ ) in addition to normal rates of inflation.

After a preliminary trial in 1986, standard therapy for $\mathrm{HCV}$ consisted of subcutaneous injections of 3 million units of interferon- $\alpha$ three times a week for 24 weeks, ${ }^{10}$ but subsequent optimization of treatment recommended the extension of therapy duration to 48 weeks in some patients and the addition of ribavirin $800 \mathrm{mg} /$ day. ${ }^{10,11} \mathrm{In}$ an economic evaluation of randomized controlled trials of

Hospital discharges coded to cirrhosis is increasing

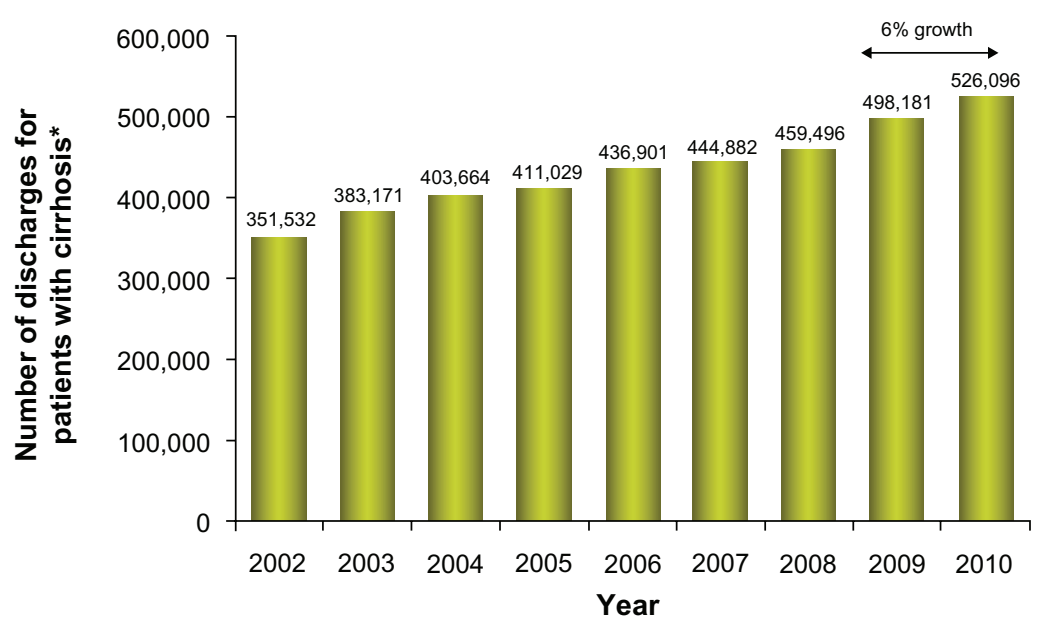

Figure I Trends in hospital discharges and in-hospital cost for cirrhosis from 2002 to 2010.

Notes: *ICD-9-CM diagnosis codes 571.2, 57I.5, 57I.6; all listed diagnoses. In general, the number of hospital discharges for cirrhosis (International Classification of Diseases, Clinical Modification, Ninth Edition codes 57I.0-57I.9) tended to increase from 1993 to 2010. Discharges were associated with a disproportionately large increase in inhospital charges. Data with permission from HCUPnet. ${ }^{71}$ 
patients with $\mathrm{HCV}$, the net cost of interferon- $\alpha$ therapy was $\$ 1827.48$ and $\$ 3654.96$ for 24 and 48 weeks of therapy, respectively. ${ }^{12}$ When combined with ribavirin, the net cost of 48 weeks of therapy substantially increased to approximately $\$ 13,885.08$. These more effective and more costly treatment regimens may allow more patients to survive to undergo liver transplantation. Economically, this translates into a greater number of patients who require medical care and incur the substantial cost of liver transplantation and follow-up management.

The present standard of care, direct antiviral agents telaprevir and boceprevir, has offered higher sustained virological response rates but with a substantial increase in cost. ${ }^{13}$ For instance, telaprevir tends to cost $\$ 49,200$ for the entire course of treatment, 12 weeks of therapy. Boceprevir costs are between $\$ 31,000$ and $\$ 53,000$ for $24-44$ weeks of therapy. ${ }^{14}$ Important to note is that these financial numbers do not include the cost of peginterferon- $\alpha$ or ribavirin.

In addition, patients awaiting transplantation must be evaluated and treated for cirrhosis-related complications to maintain overall health and be candidates for liver transplantation. ${ }^{4}$ Maintenance of these patients represents a significant health care cost; the average annual cost for treatment of decompensated cirrhosis patients is substantially higher than that of patients with compensated cirrhosis. ${ }^{15}$ Thus, cost-effective management of cirrhosis-related complications should be an economic priority.

Lastly, at a time of global economic crisis, the medical community must become more cognizant of the costeffectiveness of any drug. Recently, two studies reported on telaprevir and boceprevir therapy in treatment-naïve patients and those whose condition had relapsed or not responded to previous treatment. ${ }^{16,17}$ The marked increase in sustained virological response has shown both drugs combined with pegylated interferon and ribavirin are cost-effective when considering the decrease in lifetime incidence of liver complications, quality-adjusted life years, and the incremental cost-effectiveness ratio. ${ }^{18,19}$

\section{Economic burden of cirrhosis- related complications}

Cirrhosis remains clinically "silent" in approximately $40 \%$ of patients and becomes apparent only through routine medical examination or complaints of diffuse nonspecific symptoms (eg, anorexia, weakness, fatigue). ${ }^{1}$ These patients are said to have compensated cirrhosis and have a 5-year survival rate of approximately $90 \% .{ }^{20}$ However, compensated cirrhosis may deteriorate into a decompensated state, resulting in PH, EVB, SBP, or HE. ${ }^{1}$ Without proper management of these complications and subsequent liver transplantation, $<50 \%$ of patients with decompensated cirrhosis survive for 5 years. $^{20}$

$\mathrm{PH}$, which is often the first cause for hospitalization in patients with cirrhosis, may contribute to the development of other complications, including ascites, hepatorenal syndrome, variceal bleeding, and HE because of sinusoidal hypertension, peripheral vasodilatation, and the development of portosystemic collaterals. ${ }^{2}$ According to HCUP, the total number of hospital discharges receiving a principal diagnosis of PH or EVB has tended to decrease since $1993 .{ }^{9}$ In contrast, the number of hospital discharges with cirrhosis-related HE and SBP has increased.

Interestingly, hospital charges associated with cirrhosisrelated complications have increased from 1993 to 2009, despite either stability or reductions in the mean length of hospital stay. ${ }^{9}$ For example, mean cost associated with $\mathrm{PH}$ in 1993 was approximately $\$ 19,872$ versus $\$ 47,231$ in 2009, although the average length of hospital stay was 7.3 and 5.3 days, respectively. Similarly, in-hospital charges associated with HE have increased from \$11,808 in 1993 to $\$ 35,875$ in 2009 despite a reduction in the mean length of hospital stay ( 8.1 days in 1993 versus 5.8 days in 2009). These estimates of hospital cost associated with cirrhosis-related complications may be skewed because patients with these disorders are likely to require more than one hospital stay per year; however, the actual cost burden is probably greater than the numbers reported by HCUP because of costs associated with nonhospital care (ie, physician office visits, medications, screening for additional complications or deterioration of complications, and use of nonhospital caregiving facilities). Indeed, the percentage of patients with HE who were discharged to nursing homes or rehabilitation centers increased from $2.6 \%$ to $23.3 \%$ between 1993 and 2009.

Indirect costs (eg, lost work productivity, car accidents resulting from patients with minimal $\mathrm{HE}$ ) also have an impact on the economic burden of cirrhosis-related complications and are, to date, unexamined. Further, the cost of ineffective therapeutic and maintenance regimens, including expenditures associated with reductions in HRQOL, increase the economic burden of cirrhosis-related diseases. The evaluation and use of cost-effective therapeutic strategies to prevent the occurrence and halt the progression and relapse of various cirrhosis-related complications is essential in reducing the economic impact of these disorders. 
Hospital discharges associated with HE increased by $21 \%$ in 2010

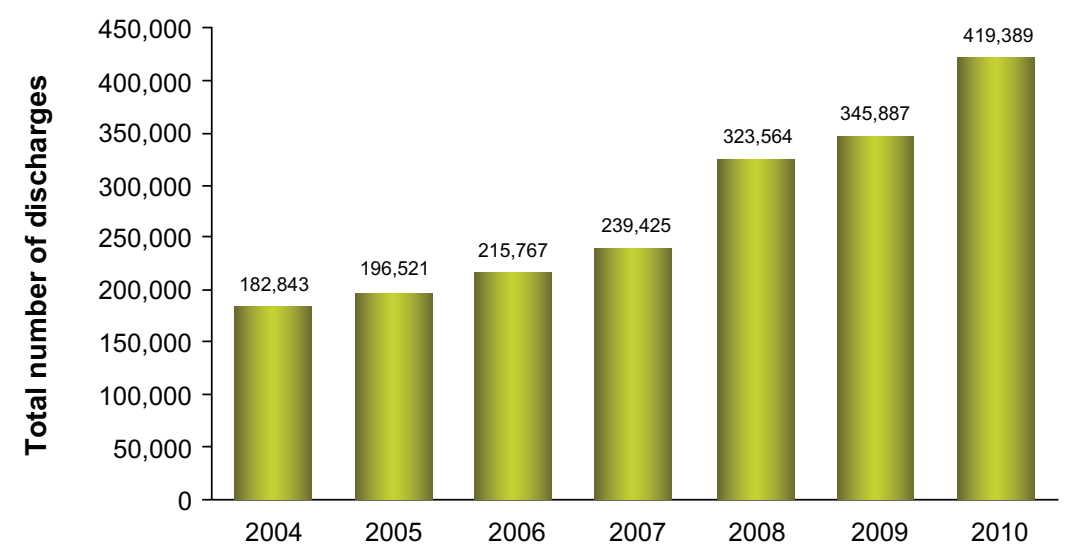

Figure 2 Increase trend in hepatic encephalopathy admissions since 2004, with 21\% during the period of 2009 to 2010 .

Notes: Data calculated using ICD-9-CM codes 291.2 (alcoholic dementia, not elsewhere classified), 348.30 (encephalopathy, not otherwise specified), and 572.2 (hepatic coma); includes all listed discharge diagnoses.

Abbreviations: HE, hepatic encephalopathy; ICD, International Classification of Diseases.

\section{Cost analysis of therapy for cirrhosis-related complications EVB}

EVB is a lethal consequence of $\mathrm{PH} .{ }^{21}$ Varices form at a rate of $8 \%$ per year in patients with cirrhosis and are associated with a hepatic venous pressure gradient $>10 \mathrm{mmHg}{ }^{22}$ According to guidelines provided by the American Association for the Study of Liver Diseases and the American College of Gastroenterology, esophagogastroduodenoscopy should be performed upon diagnosis of cirrhosis. If small varices are present but not bleeding, nonselective $\beta$-blockers may be used to prevent rupture. Patients with medium or large nonbleeding varices either may be administered $\beta$-blockers (eg, propranolol) or may have endoscopic variceal ligation (EVL) performed, depending on other diagnostic factors (eg, Child-Pugh class). Other options include surgical shunt (eg, transjugular intrahepatic portosystemic shunt [TIPS]), endoscopic sclerotherapy, although neither of these is recommended as prophylaxis for nonbleeding varices.

Primary prophylaxis with $\beta$-blocker therapy appears to be more cost-effective than EVL, sclerotherapy, shunt surgery, and "do nothing" strategies. ${ }^{21,23}$ There is some debate, however, as to whether prophylactic $\beta$-blocker therapy should be administered in patients who are at risk for variceal bleeding (eg, those with large varices, Child-Pugh class B or C cirrhosis, and presence of red wale marks upon endoscopy) or whether treatment should be given to all patients with cirrhosis, regardless of endoscopic results. In a decision model analysis, the total costs (in 2000 US dollars) associated with no prophylaxis, endoscopy screening before prophylaxis, and universal prophylaxis strategies were $\$ 36,700, \$ 37,300$, and $\$ 34,100$ per patient, respectively. ${ }^{24}$ Universal prophylaxis was associated with the highest number of quality-adjusted life years (QALYs; 6.65) compared with no prophylaxis (4.84 QALYs) and endoscopy screening (5.72 QALYs) strategies. ${ }^{24}$ These data suggest that universal prophylaxis therapy in patients with cirrhosis offers a substantial economic benefit. ${ }^{21,24}$

Once variceal bleeding occurs, antibiotics (eg, ciprofloxacin) are administered to reduce the risk of SBP or other infections in addition to pharmacologic (eg, vasopressin with or without nitroglycerin, terlipressin, somatostatin, octreotide) or endoscopic (eg, EVL, sclerotherapy) therapies to control bleeding. ${ }^{22}$ To date, no cost comparisons between these strategies (pharmacologic versus endoscopic therapy) are available, although a Cochrane metaanalysis concluded that endoscopic therapy (eg, sclerotherapy) was not superior to pharmacotherapy in patients with EVB and was associated with additional risks for adverse events versus pharmacologic intervention. ${ }^{25}$ In cases where patients' symptoms are nonresponsive to pharmacologic or endoscopic therapies, surgical techniques (eg, shunt surgery, TIPS) may be used to control variceal bleeding; however, cost comparisons of surgical techniques versus pharmacologic or endoscopic therapies have not been performed. Although surgical intervention may intuitively appear to be less cost-effective than pharmacologic or endoscopic therapies because of the occurrence of prolonged hospitalization, utilization of the intensive care unit, and perioperative blood requirements, the resulting reductions in rehospitalization and improvement in HRQOL in surgically managed patients suggest that such procedures 
should not be economically discounted. ${ }^{26}$ In addition, patients who received surgical intervention would not be required to undertake preventive measures for rebleeding, further reducing the overall cost burden. ${ }^{2}$

After initial variceal bleeding is controlled, patients who receive pharmacologic or endoscopic therapy must be actively managed to prevent recurrence. ${ }^{22}$ Nonselective $\beta$-blockers in combination with EVL are recommended, but shunt surgery or TIPS may also be implemented in cases of recurrent bleeding that do not respond to pharmacologic or endoscopic therapy. A cost analysis of observation alone, pharmacologic therapy, EVL, EVL combined with pharmacologic therapy, or TIPS demonstrated that combined treatment with $\beta$-blockers and EVL was more effective and more economical than any of the other strategies. ${ }^{27}$ In contrast, a study evaluating the cost of TIPS versus sclerotherapy or EVL demonstrated that TIPS was the most cost-effective strategy because some patients treated with sclerotherapy or EVL required subsequent TIPS due to nonresponse. ${ }^{28}$ However, this study may not have taken into account that patients receiving TIPS have a higher incidence of HE compared with those not receiving TIPS. ${ }^{2}$ Given this observation, an accurate representation of the total economic cost burden of TIPS would have to encompass the cost of treatment related to the management of HE in addition to variceal bleeding. Although no such cost analyses have been performed, it is likely that the additional cost of HE management prohibits TIPS as a first-line therapy for secondary prophylaxis.

\section{SBP}

SBP is a frequent, life-threatening complication of cirrhosis. ${ }^{29}$ This infection of ascitic fluid occurs without an intraabdominal infection or inflammation. The main causative agents of SBP, determined by culturing the ascitic fluid, are bacteria (eg, Escherichia coli, streptococci, Klebsiella spp). Patients with cirrhosis and ascites who develop symptoms of SBP (ie, fever, chills, abdominal pain, tenderness) with or without worsening of kidney or liver function may be screened for SBP, which is diagnosed when the polymorphonuclear cell count of ascitic fluid is $>250 / \mathrm{mm}^{3}$. ${ }^{2}$

Because of the bacterial origin of SBP, antibiotics (eg, cefotaxime, third-generation cephalosporins) are administered as first-line therapies and have been used as prophylaxis for SBP, although the efficacy of prophylaxis is controversial. ${ }^{30,31}$ Quinolones (eg, ciprofloxacin, norfloxacin) may be used for SBP prophylaxis in patients and may be cost-effective, especially in high-risk patients (ascitic fluid total protein $<1.5 \mathrm{~g} / \mathrm{dL}) .^{2,24,32-34}$ Universal prophylaxis in all patients with cirrhosis also may be economically favorable if the cost of the antibiotic prophylaxis is minimal. ${ }^{30}$ Unfortunately, widespread prophylaxis may elicit bacterial antibiotic resistance and reduce clinical efficacy. ${ }^{35}$ Indeed, bacterial antibiotic resistance to cefotaxime is increasing in prevalence, thus warranting the use of other antibiotics as prophylaxis and potentially impacting cost. ${ }^{36,37}$

Overall, direct cost analyses of antibiotic treatment for SBP are lacking. However, a clinical trial evaluating oral ofloxacin versus intravenous cefotaxime demonstrated comparable efficacy, disease resolution, treatment duration, and incidence of mortality. ${ }^{38}$ Given that oral ofloxacin is less expensive and does not require intravenous administration, it is more cost-effective than cefotaxime. Similar results have been reported for oral amoxicillin-clavulanic acid and ciprofloxacin versus cefotaxime. ${ }^{39,40}$

Intravenous albumin may be added as a supplement to antibiotic therapy to improve arterial blood volume and reduce the endotoxin and cytokine levels in patients. The addition of albumin to antibiotic therapy reduces the risk of renal impairment and death versus antibiotic therapy alone, but the necessity for in-hospital intravenous administration may cast doubt on the economic benefit of this strategy. ${ }^{41}$

\section{HE}

$\mathrm{HE}$ is a common condition in patients with cirrhosis, occurring in approximately $10 \%-60 \%$ of patients. ${ }^{42}$ The frequency of $\mathrm{HE}$ continues to increase, with a $21 \%$ increase in 2010 (Figure 2). In addition, the costs of HE continues to escalate to a level greater than $\$ 37,500$ per single hospitalization (Figure 3).

HE is caused by the accumulation of ammonia and other neuroactive substances within the systemic circulation due to reduced clearance by the liver as a result of portosystemic collateral formation or surgical alteration (eg, TIPS). ${ }^{43}$ This accumulation of neurotoxic substances results in altered consciousness and motor abnormalities. The disease is progressive, and the symptoms range from minimal, with patients displaying only subtle disruptions in aspects of everyday life, to clinically severe. Minimal HE occurs in at least $20 \%-60 \%$ of patients with cirrhosis and is associated with the development of overt HE; therefore, effective management of minimal HE may prevent $\mathrm{HE}$ and its associated costs (eg, hospitalization). ${ }^{42,44}$ Current treatment of HE focuses on reducing the levels of ammonia and other neuroactive substances by altering or eliminating the source of these compounds (eg, intestinal bacteria). ${ }^{43}$ 


\section{Greater than $\mathbf{5 0 \%}$ increase in cost per HE discharge since 2004}

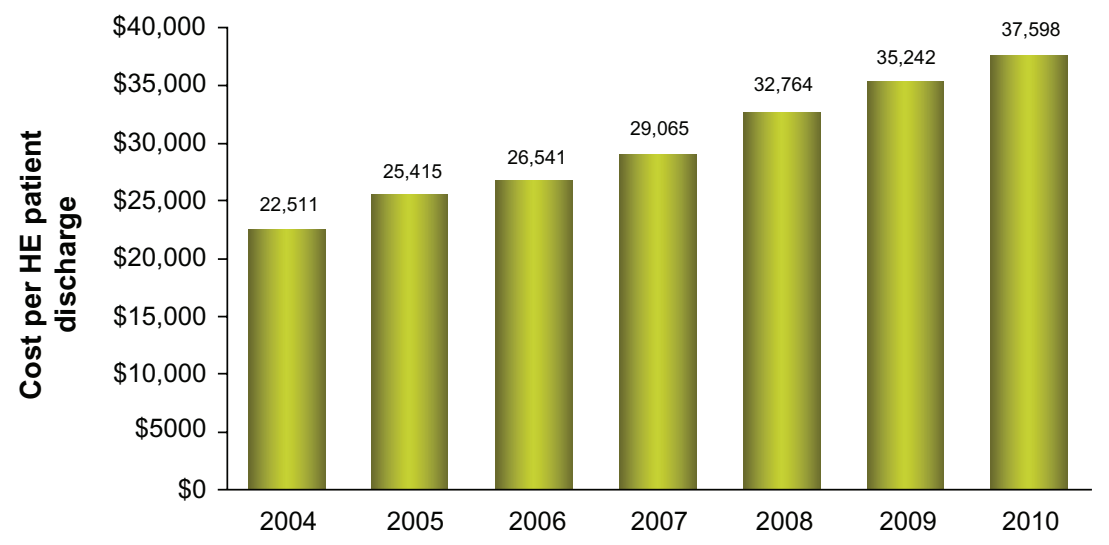

Figure 3 In-hospital cost (in US dollars) and duration of hospital stay for patients discharged with hepatic encephalopathy (International Classification of Diseases, Clinical Modification, Ninth Edition code, 572.2) or portal hypertension (International Classification of Diseases, Clinical Modification, Ninth Edition code, 572.3 ) from 2004 to 2010. Notes: *Data calculated using ICD-9-CM codes 291.2 (alcoholic dementia, not elsewhere classified), 348.30 (encephalopathy, not otherwise specified), and 572.2 (hepatic coma); includes all listed discharge diagnoses. For patients with hepatic encephalopathy or portal hypertension, the mean duration of hospital stay decreased during this period, although in-hospital charges continued to increase. Data with permission from HCUPnet. ${ }^{71}$

Abbreviations: HE, hepatic encephalopathy; ICD, International Classification of Diseases.

The standard of care for $\mathrm{HE}$ is nonabsorbable disaccharides (eg, lactulose, lactitol), which are metabolized into propionic acid and lactic acid within the colon, resulting in colonic acidification and entrapment and excretion of ammonia. ${ }^{2,43}$ Clinical evaluations of nonabsorbable disaccharides for the treatment of HE have produced contradictory results, calling into question the overall efficacy and cost-effectiveness of these therapies despite their relatively low cost. ${ }^{45-48}$ Long-term maintenance therapy with lactulose to prevent recurrence of overt episodes appears to be effective, but patient compliance may be substantially hindered by adverse events (eg, diarrhea, flatulence). ${ }^{49,50}$ Noncompliance with these therapeutic regimens would increase the possibility of HE recurrence and thus increase health care costs. There is also a lack of evidence to support the claim that nonabsorbable disaccharides prevent the progression of minimal $\mathrm{HE}$ to overt $\mathrm{HE}$, which suggests that costs associated with overt $\mathrm{HE}$, and thus treatments that perturb this progression might be more cost-effective despite a more expensive direct cost. To date, no such therapies have been identified. However, nonsystemic antibiotics may offer a beneficial long-term economic alternative to nonabsorbable disaccharides. ${ }^{50}$

Nonsystemic antibiotics (eg, neomycin, rifaximin) target the gastrointestinal flora and alter the bacterial count, thereby reducing production and accumulation of neuroactive substances. ${ }^{51}$ The efficacy of neomycin in HE alone or in combination with lactulose is questionable, ${ }^{52-57}$ and tolerability concerns ${ }^{53,55}$ prohibit its long-term use. In contrast, rifaximin alone is more effective than placebo, ${ }^{58}$ and rifaximin alone or in combination with nonabsorbable disaccharides is at least as effective as nonabsorbable disaccharides in reducing HE symptoms. ${ }^{46,54,59-64}$ In addition, rifaximin alone or in combination with nonabsorbable disaccharides alleviates HE symptoms more rapidly than lactulose, signifying the potential of rifaximin to reduce hospitalization costs. ${ }^{59,61,62,64}$

A 2007 decision model analysis examined several competing therapeutic strategies for $\mathrm{HE}$, including a "do nothing" strategy, lactulose monotherapy, lactitol monotherapy, neomycin monotherapy, rifaximin monotherapy, and rifaximin as salvage therapy in patients intolerant of lactulose. It demonstrated that the "do nothing" strategy was the least effective and rifaximin salvage therapy was the most effective ${ }^{65}$ Lactulose monotherapy and rifaximin salvage therapy were the least expensive and most effective compared with the other strategies, but rifaximin salvage therapy cost an incremental \$2315 per QALY gained versus lactulose. These findings suggest that, based on hypothetical assumptions, rifaximin therapy is more effective than no treatment but more expensive than other treatment strategies. However, overall cost-effectiveness must take into account not only the direct cost of therapy but also indirect costs, such as length of hospitalization, patient compliance or lack thereof, clinical status of patients at the end of therapy, and the potential for recurrence of an HE episode. These factors were not examined in the aforementioned study. For example, based on data from a Phase III clinical trial evaluating the efficacy of rifaximin in maintaining remission of $\mathrm{HE}$ episodes, for 
Table I Demographic and hospitalization information for both groups

\begin{tabular}{lllll}
\hline & $\begin{array}{l}\text { Gender } \\
\text { (male/ } \\
\text { female) }\end{array}$ & $\begin{array}{l}\text { MELD } \\
\text { (average) }\end{array}$ & $\begin{array}{l}\text { Age } \\
\text { (mean) } \\
\text { (years) }\end{array}$ & $\begin{array}{l}\text { Hospitalizations } \\
\text { (total) }\end{array}$ \\
\hline $\begin{array}{l}\text { Group one } \\
\text { (lactulose) }\end{array}$ & $18 / 6$ & $\begin{array}{l}14 \\
\text { (range 10-19) }\end{array}$ & 48 & 19 \\
$\begin{array}{l}\text { Group two } \\
\text { (rifaximin) }\end{array}$ & $\begin{array}{l}15 \\
\text { (range 10-18) }\end{array}$ & 47 & 3 \\
\hline
\end{tabular}

Notes: Hospitalization number and treatment cost in patients with hepatic encephalopathy who received lactulose or rifaximin therapy. Treatment with rifaximin therapy reduced the number of hospitalizations compared with treatment with lactulose therapy concurrent with a reduction in total cost of therapy per patient (2005 US dollars).

Abbreviation: MELD, Model for End-Stage Liver Disease.

every four patients treated with rifaximin, approximately one case of breakthrough HE can be prevented. ${ }^{66}$ In addition, one case of HE-related hospitalization can be prevented for every nine patients treated with rifaximin for up to 6 months. These data suggest that even though the direct cost of rifaximin is higher than placebo or "do nothing" strategies, benefits to the patient and a potential reduction in the need for long-term health care support the use of rifaximin as a cost-effective strategy.

In addition, several studies have evaluated the overall cost difference incurred by patients treated with rifaximin versus lactulose therapy ${ }^{67,68} \mathrm{~A}$ retrospective chart review, published in 2006, of patients presenting with stage II HE who received either lactulose or rifaximin therapy demonstrated that the number and duration of hospitalizations was reduced in the rifaximin group compared with the lactulose group (Table 1) ${ }^{68}$ Further, the total cost of therapy per patient per year was reduced by $\$ 5327$ with the use of rifaximin. Similar results were obtained in a 2005 retrospective chart review of patients with HE who initially received lactulose but were switched to rifaximin after its introduction in the
US in $2004 .{ }^{50}$ Compliance rate, clinical status at the end of treatment, and adverse events associated with rifaximin were also significantly more favorable than with lactulose $(P<0.001$ for all $)$ in this study. As a whole, these results imply that despite the increased direct cost of rifaximin compared with nonabsorbable disaccharides, the overall benefits of rifaximin in terms of prevention of HE-related hospitalization, breakthrough HE episodes, reduction in length of hospital stay, and increased compliance and tolerability support the economic benefit of rifaximin over nonabsorbable disaccharides.

When evaluating medical economics, all components must be considered. In the management of cirrhosis, drug costs, physician visits, procedures, and hospitalizations are the common economic components that determine the economic costs in treating the disease. HE management, in particular, rehospitalization, and length of hospital stays have been reported with economic costs recently presented. ${ }^{66}$ Decreased length of stay, time to full diet, and frequency of readmission were found in patients treated with rifaximin prior to admission (Table 2) ${ }^{69}$

As the health care system transforms into a payer system guided towards bundle reimbursements, the health care provider will need to remain cognizant of lowering hospitalization frequency and shortening length of stay. In a retrospective review presented at the 2012 American College of Gastroenterology Annual Scientific Meeting, hospital readmissions were more frequent in patients on lactulose therapies. Although overall medication costs were much higher in the rifaximin groups, hospitalizations drove up cost to a significant amount culminating in rifaximin monotherapy group overall costs being $40 \%$ less than lactulose therapy (Table 3). The primary indicators for readmission were Model for End-Stage Liver Disease-driven international normalized

Table 2 Overt hepatic encephalopathy hospitalizations identified by therapy upon admission and then in-house continuous or addition of medication

\begin{tabular}{|c|c|c|c|c|c|c|}
\hline \multicolumn{7}{|c|}{ Therapy received affected hospital stays and costs in patients with OHE } \\
\hline $\begin{array}{l}\text { Group (outpatient therapy } \rightarrow \\
\text { added hospital therapy) }\end{array}$ & $\mathbf{n}$ & $\begin{array}{l}\text { MELD score } \\
\text { (mean) }\end{array}$ & $\begin{array}{l}\text { Length of stay } \\
\text { (days) }\end{array}$ & $\begin{array}{l}\text { Time to start of full } \\
\text { diet (days) }\end{array}$ & HCUP (7,500/d) & $\begin{array}{l}\text { Insured/MC/MD } \\
\text { costs }(8,382 / d)\end{array}$ \\
\hline (I) $\mathrm{Lac} \rightarrow \mathrm{Lac}$ & 18 & 11.5 & 5.75 & 4.1 & 43,125 & 48,197 \\
\hline (2) $\mathrm{RFX} \rightarrow$ Lac & 19 & 12.5 & 3.4 & 2.25 & 25,500 & 28,329 \\
\hline (3) $\mathrm{Lac} \rightarrow \mathrm{RFX}$ & 20 & 10.5 & 4.25 & 3.5 & 31,875 & 35,624 \\
\hline (4) NT $\rightarrow$ Lac/RFX & 14 & 11.5 & 5.25 & 3.8 & 39,375 & 44,006 \\
\hline (5) $\mathrm{NT} \rightarrow \mathrm{Lac}$ & 28 & 13 & 6.5 & 4.5 & 48,750 & 54,483 \\
\hline
\end{tabular}

Notes: There were five groups of patients with three admission treatments: no treatment, lactulose, or rifaximin. Patients were then treated with lactulose, rifaximin, or both. The most expensive group in terms of length of stay and overall costs were the no treatment groups. The least expensive in terms of length of stay and overall costs was the group presenting on rifaximin therapy.

Abbreviations: d, day; HCUP, Healthcare Cost and Utilization Project; Lac, lactulose; Lac/RFX, lactulose and rifaximin combination therapy; MC, Medicare; MD, Medicaid; MELD, Model for End-Stage Liver Disease; NT, no treatment; OHE, overt hepatic encephalopathy; RFX, rifaximin monotherapy. 
Table 3

\begin{tabular}{|c|c|c|c|c|c|c|c|}
\hline Group & Group & $\begin{array}{l}\text { Medication } \\
\text { (LAC } 720 / y r \text {, } \\
\text { RIF } 13,200 / y r \text { ) }\end{array}$ & $\begin{array}{l}\text { HCUP } \\
\text { (38.5/6days) }\end{array}$ & Combined & $\begin{array}{l}\text { Total Mean } \\
\text { Costs Per } \\
\text { Patient }\end{array}$ & $\begin{array}{l}\text { Costs Range Per } \\
\text { Patient }\end{array}$ & $P$-value \\
\hline (I) LAC & (I) & 73,440 & $3,927,000$ & $4,000,440$ & 61,545 & $39,220-122,545$ & \\
\hline (2) RIF & (2) & 422,400 & 847,000 & $1,269,400$ & 39,669 & $\mid 4,609-90,450$ & 0.025 \\
\hline (3) $\mathrm{LAC}+\mathrm{RIF}$ & (3) & 570,720 & $\mathrm{I}, 50 \mathrm{I}, 500$ & $2,072,220$ & 50,542 & $26,339-105,666$ & 0.055 \\
\hline
\end{tabular}

ratio values, and an increase in Model for End-Stage Liver Disease score of 3.8 points. ${ }^{70}$

\section{Conclusion}

Despite the implementation of preventive measures, the incidence of cirrhosis has remained stable, although the health care cost associated with cirrhosis has risen. The cause of this cost increase is unknown but may be related to the cost differential between previous therapeutic regimens and new, more beneficial therapies. These more beneficial therapies, in addition to being associated with a higher direct cost, may allow a greater percentage of patients to survive to receive liver transplantation, itself a costly medical procedure requiring hospitalization and posttransplantation outpatient care. These new therapies may also increase the number of patients who will develop and require management for cirrhosis-related complications while awaiting liver transplantation. Because of this, cost-effective management of cirrhosis-related complications is essential. Analyses of drug efficacy and patient compliance and tolerability, in addition to direct costs of treatment regimens, are essential for accurate determination of the cost benefit of individual therapies; however, to date, few adequately designed studies have undertaken such analyses. Further, studies evaluating the overall cost burden, including screening costs, cost of treatment and maintenance therapy, conveyance to liver transplantation, liver transplantation success, and the economic aspect of HRQOL after transplantation, are essential for evaluation of the economic burden of cirrhosis-related complications. Until these data are assessed, it is difficult to determine the overall economic burden of cirrhosis-related complications and, more importantly, provide the most economical and beneficial treatment strategies to patients.

\section{Disclosure}

The authors report no conflicts of interest in this work.

\section{References}

1. Heidelbaugh JJ, Bruderly M. Cirrhosis and chronic liver failure: part I. Diagnosis and evaluation. Am Fam Physician. 2006;74(5):756-762.
2. Garcia-Tsao G, Lim JK. Management and treatment of patients with cirrhosis and portal hypertension: recommendations from the Department of Veterans Affairs Hepatitis C Resource Center Program and the National Hepatitis C Program. Am J Gastroenterol. 2009;104(7):1802-1829.

3. Schuppan D, Afdhal NH. Liver cirrhosis. Lancet. 2008;371(9615): 838-851.

4. Cardenas A, Gines P. Management of complications of cirrhosis in patients awaiting liver transplantation. $J$ Hepatol. 2005;42(Suppl 1): S124-S133.

5. Lim YS, Kim WR. The global impact of hepatic fibrosis and end-stage liver disease. Clin Liver Dis. 2008;12(4):733-746, vii.

6. Wasley A, Grytdal S, Gallagher K; Centers for Disease Control and Prevention. Surveillance for acute viral hepatitis - United States, 2006. MMWR Surveill Summ. 2008;57(2):1-24.

7. Lavanchy D. The global burden of hepatitis C. Liver Int. 2009;29(Suppl 1): 74-81.

8. Pyenson B, Fitch K, Iwasaki K. Consequences of Hepatitis C Virus (HCV): Costs of a Baby Boomer Epidemic of Liver Disease. New York, NY: Milliman, Inc; 2009. Available from: http://publications. milliman.com/research/health-rr/pdfs/consequences-hepatitis-c-virusRR05-18-09.pdf. Accessed January 18, 2011.

9. Lecuyer A, Levy C, Gaudelus J, et al. Hospitalization of newborns and young infants for chickenpox in France. Eur J Pediatr. 2010;169(10):1293-1297.

10. Vrolijk JM, de Knegt RJ, Veldt BJ, Orlent H, Schalm SW. The treatment of hepatitis C: history, presence and future. Neth J Med. 2004; 62(3):76-82.

11. Dienstag JL, McHutchison JG. American Gastroenterological Association technical review on the management of hepatitis C. Gastroenterology. 2006;130(1):231-264.

12. Shepherd J, Waugh N, Hewitson P. Combination therapy (interferon alfa and ribavirin) in the treatment of chronic hepatitis $\mathrm{C}$ : a rapid and systematic review. Health Technol Assess. 2000;4(33):1-67.

13. Shiffman ML, Esteban R. Triple therapy for HCV genotype 1 infection: telaprevir or boceprevir? Liver Int. 2012;32(Suppl 1):54-60.

14. McQueen C. FDA approves Vertex's new drug Incivek for treatment of hepatitis C [webpage on the Internet]. Princeton, NJ: The AIDS Beacon; May 23, 2011. Available from: http://www.aidsbeacon.com/ news/2011/05/23/fda-approves-vertexs-new-drug-incivek-telaprevirfor-treatment-of-hepatitis-c/. Accessed January 21, 2013.

15. Brown RE, De Cock E, Colin X, Antonanzas F, Iloeje UH. Hepatitis B management costs in France, Italy, Spain, and the United Kingdom. J Clin Gastroenterol. 2004;38(10 Suppl 3):S169-S174.

16. Deniz B, Brogan AJ, Miller JD, Talbird SE, Thompson JR. Projections using decision-analytic modeling of long-term clinical value of telaprevir for the treatment of HCV patients who had failed prior peginterferon/ribavirin treatment [abstract]. Hepatology. 2011;54(S1): 802A-803A.

17. Camma C, Petta S, Enea M, et al. Cost-effectiveness of boceprevir or telaprevir for untreated patients with genotype 1 chronic hepatitis $\mathrm{C}$. Hepatology. 2012;56(3):850-860.

18. Chhatwal J, Ferrante SA, Dasbach EJ, et al. Cost-effectiveness of boceprevir use in patients with chronic hepatitis $\mathrm{C}$ genotype-1 who failed prior treatment with peginterferon/ribavirin [abstract]. Hepatology. 2011;54(Suppl 1):801A-802A. 
19. Ferrante SA, Chhatwal J, Elbasha E, et al. Cost-effectiveness of boceprevir based regimens in previously untreated adult subjects with chronic hepatitis C genotype 1 [abstract]. Hepatology. 2011;54(Suppl 1):795A-796A.

20. Grewal P, Martin P. Care of the cirrhotic patient. Clin Liver Dis. 2009; 13(2):331-340.

21. Arguedas MR, Heudebert GR, Eloubeidi MA, Abrams GA, Fallon MB. Cost-effectiveness of screening, surveillance, and primary prophylaxis strategies for esophageal varices. Am J Gastroenterol. 2002;97(9):2441-2452.

22. Garcia-Tsao G, Sanyal AJ, Grace ND, Carey W. Prevention and management of gastroesophageal varices and variceal hemorrhage in cirrhosis Hepatology. 2007;46(3):922-938.

23. Teran JC, Imperiale TF, Mullen KD, Tavill AS, McCullough AJ. Primary prophylaxis of variceal bleeding in cirrhosis: a cost-effectiveness analysis. Gastroenterology. 1997;112(2):473-482.

24. Saab S, DeRosa V, Nieto J, Durazo F, Han S, Roth B. Costs and clinical outcomes of primary prophylaxis of variceal bleeding in patients with hepatic cirrhosis: a decision analytic model. Am J Gastroenterol. 2003;98(4):763-770.

25. D’Amico G, Pietrosi G, Tarantino I, Pagliaro L. Emergency sclerotherapy versus medical interventions for bleeding oesophageal varices in cirrhotic patients. Cochrane Database Syst Rev. 2002;1: CD002233.

26. O’Donnell TF Jr, Gembarowicz RM, Callow AD, Pauker SG, Kelly JJ, Deterling RA. The economic impact of acute variceal bleeding: costeffectiveness implications for medical and surgical therapy. Surgery. 1980;88(5):693-701.

27. Rubenstein JH, Eisen GM, Inadomi JM. A cost-utility analysis of secondary prophylaxis for variceal hemorrhage. Am J Gastroenterol. 2004;99(7):1274-1288.

28. Russo MW, Zacks SL, Sandler RS, Brown RS. Cost-effectiveness analysis of transjugular intrahepatic portosystemic shunt (TIPS) versus endoscopic therapy for the prevention of recurrent esophageal variceal bleeding. Hepatology. 2000;31(2):358-363.

29. Ribeiro TC, Chebli JM, Kondo M, Gaburri PD, Chebli LA, Feldner AC. Spontaneous bacterial peritonitis: how to deal with this life-threatening cirrhosis complication? Ther Clin Risk Manag. 2008;4(5):919-925.

30. Das A. A cost analysis of long term antibiotic prophylaxis for spontaneous bacterial peritonitis in cirrhosis. Am J Gastroenterol. 1998; 93(10):1895-1900.

31. Sabat M, Kolle L, Soriano G, et al. Parenteral antibiotic prophylaxis of bacterial infections does not improve cost-efficacy of oral norfloxacin in cirrhotic patients with gastrointestinal bleeding. Am J Gastroenterol. 1998;93(12):2457-2462.

32. Loomba R, Wesley R, Bain A, Csako G, Pucino F. Role of fluoroquinolones in the primary prophylaxis of spontaneous bacterial peritonitis: meta-analysis. Clin Gastroenterol Hepatol. 2009;7(4):487-493.

33. Fernandez J, Ruiz del Arbol L, Gomez C, et al. Norfloxacin vs ceftriaxone in the prophylaxis of infections in patients with advanced cirrhosis and hemorrhage. Gastroenterology. 2006;131(4):1049-1056.

34. Soriano G, Guarner C, Tomas A, et al. Norfloxacin prevents bacterial infection in cirrhotics with gastrointestinal hemorrhage. Gastroenterology 1992;103(4):1267-1272.

35. Dupeyron C, Mangeney N, Sedrati L, Campillo B, Fouet P, Leluan G. Rapid emergence of quinolone resistance in cirrhotic patients treated with norfloxacin to prevent spontaneous bacterial peritonitis. Antimicrob Agents Chemother. 1994;38(2):340-344.

36. Umgelter A, Reindl W, Miedaner M, Schmid RM, Huber W. Failure of current antibiotic first-line regimens and mortality in hospitalized patients with spontaneous bacterial peritonitis. Infection. 2009; 37(1):2-8.

37. Yakar T, Guclu M, Serin E, Aliskan H, Husamettin E. A recent evaluation of empirical cephalosporin treatment and antibiotic resistance of changing bacterial profiles in spontaneous bacterial peritonitis. Dig Dis Sci. 2010;55(4):1149-1154.
38. Navasa M, Follo A, Llovet JM, et al. Randomized, comparative study of oral ofloxacin versus intravenous cefotaxime in spontaneous bacterial peritonitis. Gastroenterology. 1996;111(4):1011-1017.

39. Ricart E, Soriano G, Novella MT, et al. Amoxicillin-clavulanic acid versus cefotaxime in the therapy of bacterial infections in cirrhotic patients. J Hepatol. 2000;32(4):596-602.

40. Tuncer I, Topcu N, Durmus A, Turkdogan MK. Oral ciprofloxacin versus intravenous cefotaxime and ceftriaxone in the treatment of spontaneous bacterial peritonitis. Hepatogastroenterology. 2003;50(53): $1426-1430$

41. Sort P, Navasa M, Arroyo V, et al. Effect of intravenous albumin on renal impairment and mortality in patients with cirrhosis and spontaneous bacterial peritonitis. N Engl J Med. 1999;341(6):403-409.

42. Poordad FF. The burden of hepatic encephalopathy. Aliment Pharmacol Ther. 2007;25(Supp1 1):3-9.

43. Gerber T, Schomerus H. Hepatic encephalopathy in liver cirrhosis: pathogenesis, diagnosis and management. Drugs. 2000;60(6): 1353-1370.

44. Hartmann IJ, Groeneweg M, Quero JC, et al. The prognostic significance of subclinical hepatic encephalopathy. Am J Gastroenterol. 2000;95(8):2029-2034.

45. Als-Nielsen B, Gluud LL, Gluud C. Non-absorbable disaccharides for hepatic encephalopathy: systematic review of randomised trials. BMJ. 2004;328(7447):1046.

46. Paik YH, Lee KS, Han KH, et al. Comparison of rifaximin and lactulose for the treatment of hepatic encephalopathy: a prospective randomized study. Yonsei Med J. 2005;46(3):399-407.

47. Poo JL, Gongora J, Sanchez-Avila F, et al. Efficacy of oral L-ornithineL-aspartate in cirrhotic patients with hyperammonemic hepatic encephalopathy. Results of a randomized, lactulose-controlled study. Ann Hepatol. 2006;5(4):281-288.

48. Sharma P, Agrawal A, Sharma BC, Sarin SK. Prophylaxis of hepatic encephalopathy in acute variceal bleed: a randomized controlled trial of lactulose versus no lactulose. J Gastroenterol Hepatol. 2011; 26(6):996-1003.

49. Sharma BC, Sharma P, Agrawal A, Sarin SK. Secondary prophylaxis of hepatic encephalopathy: an open-label randomized controlled trial of lactulose versus placebo. Gastroenterology. 2009;137(3):885-891.

50. Leevy CB, Phillips JA. Hospitalizations during the use of rifaximin versus lactulose for the treatment of hepatic encephalopathy. Dig Dis Sci. 2007;52(3):737-741.

51. Abou-Assi S, Vlahcevic ZR. Hepatic encephalopathy. Metabolic consequence of cirrhosis often is reversible. Postgrad Med. 2001; 109(2):52-54.

52. Atterbury CE, Maddrey WC, Conn HO. Neomycin-sorbitol and lactulose in the treatment of acute portal-systemic encephalopathy. A controlled, double-blind clinical trial. Am J Dig Dis. 1978;23(5):398-406.

53. Blanc P, Daures JP, Liautard J, et al. [Lactulose-neomycin combination versus placebo in the treatment of acute hepatic encephalopathy. Results of a randomized controlled trial]. Gastroenterol Clin Biol. 1994;18(12):1063-1068. French.

54. Conn HO, Leevy CM, Vlahcevic ZR, et al. Comparison of lactulose and neomycin in the treatment of chronic portal-systemic encephalopathy. A double blind controlled trial. Gastroenterology. 1977;72(4 Pt 1): 573-583.

55. Dawson AM, McLaren J, Sherlock S. Neomycin in the treatment of hepatic coma. Lancet. 1957;273(7008):1262-1268.

56. Rothenberg ME, Keeffe EB. Antibiotics in the management of hepatic encephalopathy: an evidence-based review. Rev Gastroenterol Disord. 2005;5(Suppl 3):26-35.

57. Strauss E, Tramote R, Silva EP, et al. Double-blind randomized clinical trial comparing neomycin and placebo in the treatment of exogenous hepatic encephalopathy. Hepatogastroenterology. 1992;39(6):542-545.

58. Sidhu SS, Goyal O, Mishra BP, Sood A, Chhina RS, Soni RK. Rifaximin improves psychometric performance and health-related quality of life in patients with minimal hepatic encephalopathy (the RIME Trial). Am J Gastroenterol. 2011;106(2):307-316. 
59. Bucci L, Palmieri GC. Double-blind, double-dummy comparison between treatment with rifaximin and lactulose in patients with medium to severe degree hepatic encephalopathy. Curr Med Res Opin. 1993;13(2):109-118.

60. Festi D, Mazzella G, Orsini M, et al. Rifaximin in the treatment of chronic hepatic encephalopathy: results of a multicenter study of efficacy and safety. Curr Ther Res. 1993;54(5):598-609.

61. Giacomo F, Francesco A, Michele N, Oronzo S, Antonella F. Rifaximin in the treatment of hepatic encephalopathy. Eur J Clin Res. $1993 ; 4: 57-66$.

62. Loguercio C, Federico A, De Girolamo V, Ferrieri A, Del Vecchio Blanco C. Cyclic treatment of chronic hepatic encephalopathy with rifaximin. Results of a double-blind clinical study. Minerva Gastroenterol Dietol. 2003;49(1):53-62.

63. Mas A, Rodes J, Sunyer L, et al. Comparison of rifaximin and lactitol in the treatment of acute hepatic encephalopathy: results of a randomized, double-blind, double-dummy, controlled clinical trial. J Hepatol. 2003;38(1):51-58.

64. Massa P, Vallerino E, Dodero M. Treatment of hepatic encephalopathy with rifaximin: double-blind, double dummy study versus lactulose. Eur J Clin Res. 1993;4:7-18.
65. Huang E, Esrailian E, Spiegel BM. The cost-effectiveness and budget impact of competing therapies in hepatic encephalopathy - a decision analysis. Aliment Pharmacol Ther. 2007;26(8):1147-1161.

66. Bass NM, Mullen KD, Sanyal A, et al. Rifaximin treatment in hepatic encephalopathy. $N$ Engl J Med. 2010;362(12):1071-1081.

67. Leevy CB. Economic impact of treatment options for hepatic encephalopathy. Semin Liver Dis. 2007;27(Suppl 2):26-31.

68. Neff GW, Kemmer N, Zacharias VC, et al. Analysis of hospitalizations comparing rifaximin versus lactulose in the management of hepatic encephalopathy. Transplant Proc. 2006;38(10):3552-3555.

69. Neff GW, Kemmer N, Parkinson E, et al. Outcomes in length of hospital stay in cirrhotics admitted for overt hepatic encephalopathy [abstract]. Am Coll Gastroeneterol. 2012;107(Suppl):S601.

70. Neff GW, Kemmer N, Parkinson E, et al. Readmission rates and maintenance overt hepatic encephalopathy [abstract]. Am Coll Gastroeneterol. 2012;107(Suppl 1):S184-S185.

71. Healthcare Cost and Utilization Project. Agency for Healthcare Research and Quality, [homepage on the Internet]. http://hcupnet.ahrq.gov. Accessed June 20, 2012.
ClinicoEconomics and Outcomes Research

\section{Publish your work in this journal}

ClinicoEconomics \& Outcomes Research is an international, peerreviewed open-access journal focusing on Health Technology Assessment, Pharmacoeconomics and Outcomes Research in the areas of diagnosis, medical devices, and clinical, surgical and pharmacological intervention. The economic impact of health policy and health systems

\section{Dovepress}

organization also constitute important areas of coverage. The manuscript management system is completely online and includes a very quick and fair peer-review system, which is all easy to use. Visit http://www.dovepress.com/testimonials.php to read real quotes from published authors. 